\title{
Comment on: Trans-Tympanic Cartilage Chip Insertion for Intractable Patulous Eustachian Tube
}

\author{
Michael JO Boedts ${ }^{1,2}$ \\ ${ }^{1}$ Brain Research Center for Advanced, Innovative, Interdisciplinary and International Neuromodulation (Brai3n), Ghent, Belgium \\ ${ }^{2}$ AZ Maria Middelares, Ghent, Belgium
}

\section{Dear Editor,}

We read the report by Jeong, et al. [1], of this promising new technique with great interest. We wonder wether the application of a patch, after having inserted the cartilage, might be a confounding factor. Application of a paper patch, left on the tympanic membrane for about a month without any adjunctive therapy, effectively eliminated autophony; temporarily in $76.2 \%$, and definitely in $50 \%$, in a series of unselected autophony patients [2].

This paper patching treatment is actually a form of mass loading of the tympanic membrane. Since the inclusion criteria in the present series included a positive response to mass loading, one would expect paper patching in the present selected series to give excellent results; temporarily in almost $100 \%$ and definitely in a great majority.

A possible way to find out whether part of the results could be the result of the patching only, would be to scan the patient files for the complaint of fullness feeling. Indeed paper patching is only effective when autophony is accompanied by fullness feeling.

\section{Conflicts of interest}

The author has no financial conflicts of interest.

\section{ORCID iDs}

Michael JO Boedts https://orcid.org/0000-0001-5018-2306

\section{REFERENCES}

1) Jeong J, Nam J, Han SJ, Shin SH, Hwang K, Moon IS. Trans-tympanic cartilage chip insertion for intractable patulous eustachian tube. J Audiol Otol 2018;22:154-9.

2) Boedts M. Paper patching of the tympanic membrane as a symptomatic treatment for patulous eustachian tube syndrome. J Laryngol Otol 2014;128:228-35.

Received December 16, 2018 / Accepted December 31, 2018

\section{Address for correspondence}

Michael JO Boedts, MD

Brain Research Center for Advanced, Innovative, Interdisciplinary and International Neuromodulation (Brai3n), Jemappesstraat 5, Ghent 9000, Belgium

Tel+32-468340844 / E-mail Michael.boedts@brai3n.com

This is an Open Access article distributed under the terms of the Creative Commons Attribution Non-Commercial License (https://creativecommons.org/licenses/by-nc/4.0/) which permits unrestricted non-commercial use, distribution, and reproduction in any medium, provided the original work is properly cited. 


\section{Response}

\author{
Junhui Jeong ${ }^{1}$ and $\ln$ Seok Moon ${ }^{2}$ \\ ${ }^{1}$ Department of Otorhinolaryngology, National Health Insurance \\ Service Ilsan Hospital, Goyang, Korea \\ ${ }^{2}$ Department of Otorhinolaryngology, Yonsei University College of \\ Medicine, Seoul, Korea
}

\section{To the Editor:}

We read the comment on our manuscript with great interest. We appreciate the author's comment and would like to clearly explain our method.

\section{Effects of paper patching were negligible}

The author mentioned that application of a paper patch after cartilage chip insertion might be a confounding factor. As the author commented, the author [1] reported that paperpatching only of patulous Eustachian tubes (PETs) improved PET symptoms temporarily in $76.2 \%$ and permanently in $50 \%$ of patients. We acknowledge that paper-patching imposes a mass loading on the tympanic membrane; thus, patching per se could improve the symptoms of autophony. Recently, Kim, et al. [2] reported that repetitive paper-patching alleviated aural discomfort in most PET patients for at least 3 months and they suggested that it could serve as a first-line treatment for PET.

However, there are several differences between the cited studies and our work. The author [1] applied three patches on each occasion; we placed only one. Both the author [1] and Kim, et al. [2] repeated paper-patching if a patch became detached or drum immobilization was inadequate; we did not repeat patching. Our objective was to allow the incised drum to heal well, not to immobilize the tympanic membrane. Thus, we removed the patch after 1 week (during outpatient follow-up); no autophony symptoms developed after patch removal and all patients improved immediately. A chitin sheet (similar to a paper patch) promoting closure of the incision in the tympanic membrane has been placed after trans-tympanic silicone plug insertion in other studies, but the effects of patching on PET symptoms have not been discussed [3-5].

In our study, autophony symptoms improved immediately after surgery in $92.9 \%$ of operated ears and such improvements persisted after patch removal. The improvement rate fell to $64.3 \%$ (because of symptom redevelopment) at the last visit (after more than 12 months of follow-up). We suggest that symptom recurrence was not attributable to paperpatching, the effects of which were negligible.

\section{Our patients exhibited more symptoms of autophony than aural fullness}

The author mentioned that paper-patching was most effective for patients with both autophony and a sense of aural fullness; we evaluated the effects of patching on aural fullness. The mean aural fullness symptom scores in patients with PETs were all lower before and after surgery and at follow-up than the voice echoing/breathing scores (indicative of autophony). Although many patients exhibited autophony symptoms in the absence of aural fullness, $92.9 \%$ of ears improved in the immediate postoperative period after cartilage chip insertion with paper-patching. This was not as might be predicted by the author. Thus, we consider that any effects of paper-patching in our study were minor.

In summary, although the effects of paper-patching on a PET must certainly be considered, we found that any effect was minor/negligible, indeed less than the effects of the inserted cartilage chip. We hope that we have now adequately clarified our methodology.

\section{REFERENCES}

1) Boedts M. Paper patching of the tympanic membrane as a symptomatic treatment for patulous eustachian tube syndrome. J Laryngol Otol 2014;128:228-35

2) Kim SJ, Shin SA, Lee HY, Park YH. Paper patching for patulous eustachian tube. Acta Otolaryngol 2019;139:122-8.

3) Sato T, Kawase T, Yano H, Suetake M, Kobayashi T. Trans-tympanic silicone plug insertion for chronic patulous Eustachian tube. Acta Otolaryngol 2005;125:1158-63.

4) Endo S, Mizuta K, Takahashi G, Nakanishi H, Yamatodani T, Misawa $\mathrm{K}$, et al. The effect of ventilation tube insertion or trans-tympanic silicone plug insertion on a patulous Eustachian tube. Acta Otolaryngol 2016;136:551-5.

5) Kikuchi T, Ikeda R, Oshima H, Takata I, Kawase T, Oshima T, et al. Effectiveness of Kobayashi plug for 252 ears with chronic patulous Eustachian tube. Acta Otolaryngol 2017;137:253-8. 